\title{
Pays-Bas. La nouvelle autonomie des établissements scolaires
}

Études de cas

Hetty Mulder

\section{CpenEdition}

\section{Journals}

Édition électronique

URL : https://journals.openedition.org/ries/1291

DOI : 10.4000/ries. 1291

ISSN : 2261-4265

Éditeur

France Education international

Édition imprimée

Date de publication : 1 décembre 2005

Pagination : 71-74

ISSN : $1254-4590$

Référence électronique

Hetty Mulder, "Pays-Bas. La nouvelle autonomie des établissements scolaires », Revue internationale d'éducation de Sèvres [En ligne], 40 | décembre 2005, mis en ligne le 17 novembre 2011, consulté le 09 juillet 2021. URL : http://journals.openedition.org/ries/1291 ; DOI : https://doi.org/10.4000/ries.1291

Ce document a été généré automatiquement le 9 juillet 2021

(C) Tous droits réservés 


\section{Pays-Bas. La nouvelle autonomie des établissements scolaires ${ }^{1}$}

Études de cas

Hetty Mulder

1 Au cours de ces dernières années, la législation néerlandaise, pléthorique et surchargée de détails, a empêché les établissements du premier cycle de l'enseignement secondaire d'atteindre les objectifs qu'ils s'étaient eux-mêmes fixés. Il est devenu de plus en plus difficile de gérer l'hétérogénéité des élèves. La recherche d'une cohérence des curricula est devenue la dernière des priorités.

\section{L'émergence de l'autonomie}

2 Le temps est venu maintenant de bénéficier d'une plus grande autonomie et de revoir à la hausse nos attentes. Inutile désormais de chercher le moteur de notre développement dans la législation; mieux vaut faire appel à notre discernement professionnel ainsi qu'aux moyens et aux aspirations des établissements et des enseignants. Cela suppose une plus grande autonomie pour diversifier l'offre éducative selon des caractéristiques générales, la formulation d'un ensemble d'objectifs communs et fondamentaux et la définition d'un certain nombre de conditions de développement : une dynamique centrée sur l'élève, une plus grande cohérence des curricula et un intérêt pour les processus d'apprentissage progressifs.

Dans le premier cycle de l'enseignement secondaire, on est passé en 1993 d'un curriculum national entièrement encadré, avec un nombre obligatoire d'heures d'enseignement par discipline, à un tronc commun préétabli et à de simples recommandations en matière d'aménagement du temps scolaire. Une plus grande autonomie a été accordée aux établissements afin qu'ils déterminent leur propre curriculum et aménagent eux-mêmes le temps scolaire, leur permettant ainsi d'atteindre les objectifs fondamentaux qu'ils s'étaient fixés avec leurs élèves. 
4 L'objectif du nouveau tronc commun était probablement d'accorder une plus grande liberté aux établissements pour qu'ils définissent leur propre politique. Dans les faits, les choses se sont avérées tout autres. Pour commencer, la transition semble avoir été plus difficile que prévu. Les incitations au changement ont d'abord fait l'objet de débats législatifs. La technologie et le savoir vivre en société sont devenus des matières séparées et obligatoires. Des connaissances communes à plusieurs curricula et d'autres liées à des disciplines spécifiques ont été intégrées aux objectifs fondamentaux. On a mis en place une évaluation pour ces nouvelles connaissances, sous forme de tests obligatoires qui devaient être les mêmes pour tous les élèves ; leur notation et leur suivi administratif devaient être conformes aux recommandations officielles.

5 Six ans après avoir introduit la formation de base (Basisvorming), l'inspection scolaire a publié un rapport d'évaluation sur la mise en place du nouveau tronc commun dans les établissements. La majorité d'entre eux avait appliqué le nouveau curriculum dans ses modalités pratiques mais ne l'avait pas traduit en termes pédagogiques et méthodologiques. Aucun établissement n'avait atteint $100 \%$ de ses objectifs. Les curricula, surchargés et morcelés, avaient entraîné des difficultés, en particulier dans l'enseignement préprofessionnel. Par ailleurs, en suivant les manuels scolaires, un grand nombre d'enseignants avaient perdu toute initiative en matière de pratique pédagogique.

6 Il est temps d'améliorer les rapports entre le gouvernement et les établissements. Il s'agit d'œuvrer vers moins de règles et plus d'ambition, vers une plus grande volonté d'autonomie et une plus grande exigence en retour. Il n'est pas question de nier le rôle du gouvernement en matière d'accès à l'enseignement (secondaire) et d'évaluation de la qualité, mais il faut que ce rôle se manifeste différemment.

\section{Recommandations pour la réforme}

7 Le rapport final de la Commission pour la réforme du premier cycle de l'enseignement secondaire est le fruit de dix-huit mois d'une communication intense auprès des enseignants du premier cycle du secondaire et de la direction des établissements. On a eu recours à des grandes conférences, à des consultations à petite échelle, à des enquêtes, à des discussions formelles ou informelles et à de la correspondance postale et électronique. La Commission a consulté par ailleurs un nombre considérable d'experts et d'acteurs du système éducatif.

\section{Dans les établissements}

8 La principale recommandation au gouvernement est de donner aux établissements les pleins pouvoirs pour qu'ils définissent eux-mêmes les grandes lignes du premier cycle de l'enseignement secondaire. Il est très ambitieux d'organiser les deux premières années du secondaire de manière qu'elles fassent le lien entre l'enseignement primaire et l'enseignement secondaire supérieur, qu'il soit général ou professionnel. Cela exige des curricula sur mesure, adaptés aux caractéristiques des 12-15 ans, tenant compte des grandes disparités entre élèves et ajustés aux spécificités de chaque établissement. Le gouvernement, en fonction de son projet d'ensemble, doit définir clairement les grandes lignes de sa réforme : un curriculum plus cohérent, un rôle actif demandé aux élèves et des processus cognitifs progressifs tout au long du cursus scolaire. 
9 Pour remplir ces objectifs, le gouvernement devrait assouplir la législation sur deux points : les contenus éducatifs et l'aménagement du temps scolaire. La Commission suggère de remplacer les 260 objectifs fondamentaux actuels par 58 objectifs qui serviraient de fil conducteur au tronc commun du premier cycle de l'enseignement secondaire. Ces objectifs concerneraient au moins deux tiers des deux années de scolarité. La Commission propose par ailleurs de libéraliser le nombre d'heures d'enseignement obligatoire; actuellement fixé à 1067 heures complètes par an, ce nombre pourrait fluctuer dans une fourchette comprise entre 950 et 1150 heures. Il n'y aurait pas d'autres recommandations. La Commission préconise que soient dispensés d'une seconde langue vivante obligatoire les élèves qui devront vraisemblablement suivre une formation professionnelle de premier cycle ou qui ont besoin d'un soutien supplémentaire pour suivre cette filière.

10 La libéralisation des moyens va modifier la relation entre les établissements et le gouvernement. Les établissements pourront remanier en profondeur leur curriculum au niveau notamment des disciplines et des domaines d'apprentissage, ou encore en introduisant la pédagogie de projet. Dans la limite de leurs moyens, ils pourront définir leurs propres approches pédagogiques et méthodologiques et fixer les niveaux à atteindre par les différents groupes d'élèves. La Commission propose quatre scénarios à partir desquels les établissements peuvent déterminer le contenu des cours. Les différences entre établissements vont alors s'accentuer. C'est pourquoi le gouvernement devra rester vigilant sur l'assurance qualité. La Commission recommande au gouvernement de promouvoir et de valoriser tout d'abord l'assurance qualité en interne dans l'établissement, et en externe la qualité de sa gestion auprès des acteurs concernés. Cet aspect doit être au cœur des préoccupations du gouvernement qui exerce un droit de contrôle.

\section{Dans le domaine pédagogique}

11 Prendre des décisions qui touchent les curricula, les pratiques pédagogiques et l'orientation des élèves constituera une petite révolution pour un grand nombre d'établissements. Les enseignants devront coopérer et travailler en équipe pour que les curricula gagnent en cohérence et en qualité d'adaptation. De nouveaux environnements d'apprentissage seront nécessaires, ainsi qu'un bon équipement en technologies de l'information et de la communication. Le gouvernement devra favoriser, dans les années à venir, le développement de nouveaux outils d'apprentissage d'un usage plus souple que les outils actuels. La Commission préconise de donner plus de possibilités aux enseignants de diversifier les fonctions qu'ils exercent et de développer leurs compétences propres. Elle est favorable à ce que les enseignants débutants suivent une formation dont l'organisation pourrait être prise en charge par les établissements. Cependant, le plus important est que les enseignants disposent de suffisamment de temps. La Commission a estimé que la mise en œuvre de ces recommandations coûtera 192 euros par élève du premier cycle de l'enseignement secondaire, soit environ 80 millions d'euros par an. Afin de mener à bien ces objectifs, la Commission recommande au gouvernement de lancer un projet sur quatre ans pour le premier cycle de l'enseignement secondaire.

12 La Commission adopte en principe le point de vue de l'élève, convaincue que là réside la solution. Surcharge et morcellement sont la conséquence naturelle du mode 
d'organisation du premier cycle de l'enseignement secondaire. La solution consiste à poser un regard neuf en adoptant le point de vue de l'élève. Dans le premier cycle de l'enseignement secondaire, les élèves sont à un moment charnière de leur scolarité : ils doivent s'intégrer dans la classe, à la bonne place, tout en s'orientant dans une voie qui leur assure un avenir. Avec l'aide de ses collègues, l'enseignant(e) propose des aménagements aux différents groupes d'élèves pour y parvenir. C'est l'établissement qui a la responsabilité de mettre en œuvre un enseignement secondaire de premier cycle cohérent, adapté à son public d'élèves et d'enseignants.

\section{NOTES}

1. Ce texte est un compte rendu du rapport de justification de la Task Force for the Reform of Lower Secondary Education, publié en juin 2004.

INDEX

Index géographique : Pays-Bas

\section{AUTEUR}

\section{HETTY MULDER}

Institut national pour le développement de curricula (SLO). 